\title{
A pediatric case of Fascioliasis with eosinophilic pneumonia
}

\author{
Gülsüm İclal Bayhan¹, Abdulsamet Batur², Ayşegül Taylan-Özkan³, Kaan Demirören4, \\ Yunus Emre Beyhan ${ }^{5}$ \\ Division of ${ }^{1}$ Pediatric Infectious Disease and 4 Pediatric Gastroenterology, Deparment of Pediatrics, Department ${ }^{3}$ of \\ ${ }^{2}$ Radiology and ${ }^{5}$ Parasitology, Yuzuncu Yil University, Faculty of Medicine, Van, ${ }^{3}$ Department of Medical Microbiology, \\ Hitit University, Faculty of Medicine, Çorum, Turkey. E-mail: gibayhan@gmail.com \\ Received: 16 March 2015, Revised: 28 April 2015, Accepted: 10 June 2015
}

SUMMARY: Bayhan Gİ, Batur A, Taylan-Özkan A, Demirören K, Beyhan YE. A pediatric case of Fascioliasis with eosinophilic pneumonia. Turk J Pediatr 2016; 58: 109-112.

Fasciolia spp. are common trematode infestations worldwide. Fasciolia spp. may lead to hepatic diseases in the acute phase and may cause biliary diseases in the chronic phase. In addition, Fasciolia spp. may rarely cause extrahepatic signs and symptoms. The clinical manifestations of fascioliasis are divided into three groups: typical, atypical, and ectopic. Eosinophilic pneumonia is an atypical presentation of acute fascioliasis and it has been reported very rarely. Herein, we report a boy with marked blood eosinophilia and eosinophilic pneumonia who was diagnosed with fascioliasis by serologic tests and abdominal USG. The patient recovered completely following triclabendazole treatment.

Key words: fascioliasis, eosinophilic pneumonia, eosinophilic leukemoid reaction, child.

Fascioliasis is a zoonotic foodborne trematode infection caused by Fasciola spp. which are flat leaf-like worms known as liver flukes. The two species that infect humans are Fasciola hepatica and Fasciola gigantica ${ }^{1}$. Other most prominent liver flukes are Clonorchis sinensis, Opisthorchis felineus and Opisthorchis viverrini ${ }^{2}$. World Health Organization estimates that at least 2.4 million people are infected with fascioliasis in more than 70 countries worldwide, with several million at risk. Fasciolia spp. have worldwide distribution and not only animal cases but also human cases have been reported ${ }^{1,2}$. Humans are infected by ingestion of watercress, salad vegetables, and water which are contaminated with metacercariae ${ }^{1,3}$. Clinical spectrum of the infection is variable; however, fascioliasis cases with lung involvement are very rare. Herein, we report a rare presentation of eosinophilic pneumonia accompanied by eosinophilic leukemoid reaction in the course of fascioliasis.

\section{Case Report}

A 4-year-old boy presented with a one-month history of abdominal pain, poor appetite, and occasional fever. He had lost one kilo in the last one month and had no history of worm defecation. Physical examination was normal and the laboratory studies revealed peripheral blood leukocyte count 32,300/ $\mathrm{mm}^{3}$; hemoglobin $10.9 \mathrm{~g} / \mathrm{dl}$; platelet count $29,000 / \mathrm{mm}^{3}$; C-reactive protein (CRP) level $5.7 \mathrm{mg} / \mathrm{L}$; erythrocyte sedimentation rate 32 $\mathrm{mm} / \mathrm{h}$ with $64 \%$ eosinophils, $18 \%$ lymphocytes, $2 \%$ monocytes, and $16 \%$ polymorphonuclear leukocytes. Total immunoglobulin E level was $906 \mathrm{IU} / \mathrm{mL}$ (0-5) and the Ig G level was normal. Renal and hepatic function tests were normal. The patient had severe eosinophilia (absolute eosinophil count was $20,672 / \mathrm{mm}^{3}$ ) and was admitted to the hospital for the investigation of the etiology of eosinophilic leukemoid reaction. Parasite stool tests by "formalin ether sedimentation" and "saturated sodium chloride flotation" methods were negative three times. Echinococcus granulosus indirect hemagglutination assay (IHA) was negative but Fasciola hepatica IHA, which was repeated twice, was positive at a titer of $1 / 640$. Toxocara IgG ELISA was slightly positive (0.539; range, $0-0.500)$ at the beginning since second repetition was negative, it was considered as cross reactivity. Abdominal ultrasonography (USG) revealed a broad range of geographic style with hypoechogenicity showing predominantly periportal extension 
between the subcapsular space and the hilar region on the left lobe of the liver. This appearance was typical for fascioliasis with hepatic involvement (Fig. 1). Chest radiography showed bilateral peribronchial thickening. Thoracic computed tomography (CT) confirmed eosinophilic pneumonia that revealed diffuse bilateral nodules in the lung and peripheral nodules in lower lobes. Focal budding branches were visualized in the inferior lingula of the left lung (Fig. 2) and in the posterior lower lobe of the right lung. Echocar diogram was normal.

The patient had a history of consuming watercress. The diagnosis of eosinophilic pneumonia associated with fasciola hepatica infestation was confirmed based on the laboratory examinations, abdominal USG, and thoracic CT findings. The patient was treated with albendazole for five days. At the end of the treatment, the WBC count was $22,000 / \mathrm{mm}^{3}$ and the absolute eosinophil count was 13,200/ $\mathrm{mm}^{3}$. Following partial response to albendazole, the patient was given triclabendazole $(10 \mathrm{mg} /$ $\mathrm{kg} /$ dose) (Egaten ${ }^{\circledR}$, Novartis Pharma) for two days. The patient developed fever both on the day when treatment was started and the following two days, and then the fever dropped spontaneously. The patient was discharged five days after the completion of the triclabendazole treatment. At week 2 posttreatment, the WBC count decreased to $11,600 / \mathrm{mm}^{3}$, absolute eosinophil count was lowered to $696 / \mathrm{mm}^{3}$, and F. hepatica IHA was positive at a titer of $1 / 320$. At one month after treatment, the abdominal pain was resolved, WBC count was 8,100 / $\mathrm{mm}^{3}$, absolute eosinophils count was 486/ $\mathrm{mm}^{3}$, total IgE level decreased to $160 \mathrm{IU} / \mathrm{mL}$, F. hepatica IHA was negative, abdominal USG was completely normal, and chest X-ray was normal. Complete recovery was confirmed by these findings.

\section{Discussion}

Fascioliasis is a zoonosis caused by Fasciola hepatica or Fasciola gigantica. The most common natural hosts are sheep, cattle, and goats. The life cycle begins when the parasite's eggs in stool are deposited in water. After larvae are ingested with contaminated food or water, a symptomless incubation period starts, lasting for a few days to a few months. This is followed by an acute (hepatic) and a chronic (biliary) clinical phase. The acute phase lasts for 2-4 months and it begins when the immature worms penetrate the intestinal wall and escape into the peritoneal cavity. From here, they puncture the hepatic surface and digest and consume hepatocytes until they reach the bile ducts. This invasion kills the hepatic cells and causes varying degrees of internal bleeding. The clinical symptoms and findings detected in this stage are related to the destruction and inflammation in response to migration of the larva within the liver ${ }^{1,2,4,5}$. In the acute phase, no eggs are found in the stool. On the other hand, the chronic phase begins when the worms reach the bile ducts approximately 6 months after the ingestion of parasites. The parasites become mature on the bile ducts and start producing eggs. These eggs are released into the bile and reach the intestine, where they are found in the stool. Chronic phase may last for several years (>10 years) and eosinophilia is resolved in half of the chronic patients; therefore, absence of eosinophilia does not rule out fascioliasis $1,2,4$.

In a Fasciola hepatica case series from Turkey, abdominal pain was reported in $100 \%$, fever in $59 \%$, nausea in $14 \%$, chills $18 \%$, weight loss in $18 \%$, pruritus and urticaria in $5 \%$ of the patients with hepatic phase fascioliasis. On physical examination, mild right upper quadrant tenderness was reported in $68 \%$ and hepatomegaly in $27 \%$ of the patients. In patients with chronic phase fascioliasis, abdominal pain was reported by $100 \%$, fever in $25 \%$, nausea in $38 \%$, and weight loss in $13 \%$, upper quadrant tenderness in $63 \%$ and scleral icterus in $13 \%$ of the patients ${ }^{6}$. It was reported that, in the chronic phase, about half of the cases are asymptomatic. Common symptoms of chronic phase include intermittent pain, jaundice, and anemia ${ }^{4}$. Our patient presented with the most common symptoms of acute fascioliasis: intermittent fever, abdominal pain, and weight loss.

If the symptoms that last for $\leq 4$ months are presented with no motile echogenic changes in the gallbladder on ultrasound, the patient should be considered as acute. However, if the symptoms last for $>4$ months in conjunction with positive biliary ultrasound findings, the patient should be considered as chronic ${ }^{7}$. Our 
patient was diagnosed as acute fascioliasis since he had severe abdominal pain, fever and weight loss for one month and also had marked eosinophilia and history of consuming watercres. In USG examination, hepatic parenchymal lesions were detected while the bile ducts were normal. As expected, the stool test was negative for Fasciola spp. eggs but serology was positive by IHA.

The acute form of fascioliasis is classified into three subtypes: typical, ectopic and atypical. Typical fascioliasis has the classic triad of fever, hepatomegaly and abdominal pain. This clinical picture is called "fasciolitic hepatitis" or "febrile fasciolitic eosonophilic syndrome". Moreover, this clinical picture may be accompanied by vomiting, anorexia, weight loss, malaise, urticarial, anemia, and leukocytosis ${ }^{3}$.

Ectopic fascioliasis is the appearance of immature flukes in the tissues other than liver. The most frequently involved tissue is subcutaneous tissue ${ }^{3}$. Ectopic infections in the intestinal wall, lungs, pancreas, eye, brain, stomach wall, pharyngeal mucosa, skin and other locations are very rare $^{2}$.

F. hepatica has a special tropism to the liver ${ }^{3}$. However, some patients have symptoms related to organs other than liver. The clinical form associated with eosinophilic reaction without direct parasitic involvement accompanied by extra-hepatic organ-related symptoms and findings is called the "atypical form" 3 . In this form, the parasites are rarely found in the extrahepatic tissue, and the pathogenesis of these manifestations is suggested to be immunemediated $^{3}$. The atypical form is associated with electrocardiographic abnormalities, pericarditis, neurological symptoms including seizure, cephalgia, meningeal syndrome, and focal neurological symptoms, and altered intellectual function ${ }^{3}$. Respiratory symptoms such as cough, hemoptysis and dyspnea are uncommon and generally mild ${ }^{3}$. Parenchymal infiltrations resembling Loeffler's syndrome and pleural effusion are the most common radiologic manifestations. Both of them, especially Loeffler's syndrome, have been reported very rarely in association with the course of fascioliasis $3,8,9$. Diagnosis of atypical fascioliasis may be very difficult; however, presence of eosinophilia in the blood or other body fluids is usually the most important sign for the diagnostic process. In our patient, the hepatic findings detected on the abdominal USG were consistent with those of typical fascioliasis. On the other hand, the pulmonary findings were consistent with those of atypical form.

Eosinophilic pneumonia can be caused by other parasites aside from Fasciola spp, especially Ascaris, Strongyloides, Paragonimus, Trichinella, and Toxocara10,11. In our patient repeated stool examinations were negative for Strongyloidies stercoralis larvae and Ascaris lumbricoides ova as well as serum Toxocara IgG ELISA was negative. Trichinellosis is not common in Turkey because of the eating habit so it is not considered since there is no history of eating pork or meat from unknown source. Paragonimus infection is not seen in humans nor animals in Turkey. Also there are no eating habits of raw-shelled crabs and crustacean, so trasmission of Paragonimus spp. is not probable. Additionally, his chest $\mathrm{X}$-ray findings were resolved completely with triclabendazol treatment. Therefore, our patient was considered to have a secondary eosinophilic pneumonia to the fascioliasis.

Most common cause of eosinophilia is the presence of parasites, and the parasitic infections can induce extreme eosinophilia $\left(>5,000\right.$ cells $\left./ \mathrm{mm}^{3}\right)$ or eosinophilic leukemoid reaction $(>30 \%$ eosinophils with a WBC count of $\left.>30,000 / \mathrm{mm}^{3}\right)$. With the exception of helminthic infections, the secondary causes of eosinophilia rarely cause an absolute eosinophil count of $>1500$ cells $/ \mathrm{mm}^{3}$. The parasites which cause tissue invasion may result in extreme eosinophilia ${ }^{12}$. It is reported that the most common laboratory abnormalities of fascioliasis are the increased levels of total IgE, total IgG, leukocytosis and eosinophilia elevations in the erythrocyte sedimentation rate (ESR) ${ }^{6,7}$. Eosinophil count is variable in fascioliasis patients, particularly higher in acute cases $^{3,7,13}$. Our case was in the acute phase and was detected with marked eosinophilia and elevated IgE level. Due to the presentation of eosinophilic leukemoid reaction and the high prevalence of endemic parasitic diseases in our region, we primarily investigated parasitic diseases in our patient.

Diagnostic USG findings of fascioliasis generally reveal two types of lesions in the acute phase: one of them usually consists of multiple 
geographic hypoechoic nodules with irregular and poorly-defined margins and these nodules have peripheral and subcapsular localization, generally around the biliary tract in the liver, whereas the second type is tunnel-like branching spaces, which are also peripherally located and caused by parasite migration $7,13,14$. It was reported that, USG may not be diagnostic in the hepatic phase secondary to heterogeneity of the liver because of the poorly defined nodules; it is more useful in the biliary stage of the disease $^{15}$. In our patient, the abdominal USG findings were similar to the reported findings in previous studies; therefore, abdominal CT was not required. We consider that since tomography results in high radiation and USG can yield valuable information, USG should be the first choice in the diagnosis of fascioliasis in children. If the patient is considered to be fascioliasis, and ultrasound does not detect diagnostic findings, abdominal tomography may be taken.

The criteria for complete recovery include recovery of symptoms, resolution of peripheral eosinophilia, absence of eggs in the stool, gradual decrease in serology titers and improvement of radiologic findings ${ }^{3,5}$. In our patient, the eosinophil count decreased within two weeks and Fasciola spp. serology became negative within 45 days after treatment.

In conclusion fascioliasis should be suspected in the differential diagnosis of the patients presenting with abdominal pain, fever, weight loss, and especially with eosinophilia and elevated IgE levels. In fascioliasis, radiological examinations especially abdominal USG in acute stage are highly useful for diagnosis. Clinicians should keep in mind that fascioliasis can cause eosinophilic pneumonia.

\section{REFERENCES}

1. http://www.who.int/foodborne trematode infections/ fascioliasis/en/ (Access Date February 25, 2015)

2. Fürst T, Duthaler U, Sripa B, Utzinger J, Keiser J. Trematode infections. Infect Dis Clin North Am 2012; 26: 399-419.

3. Arjona R, Riancho JA, Aguado JM, Salesa R, GonzalezMacias J. Fascioliasis in developed countries: A review of classic and aberrant forms of the disease. Medicine (Baltimore) 1995; 74: 13-23.

4. Kaya M, Beştaş R, Cetin S. Clinical presentation and management of Fasciola hepatica infection: single-center experience. World J Gastroenterol 2011; 17: 4899-4904.
5. Graham CS, Brodie SB, Weller PF. Imported Fasciola hepatica infection in the United States and treatment with triclabendazole. Clin Infect Dis 2001; 33: 1-5.

6. Kaya M, Beştaş R, Cetin S. Clinical presentation and management of Fasciola hepatica infection: single-center experience. World J Gastroenterol 2011; 28; 17: 48994904.

7. Karahocagil MK, Akdeniz H, Sunnetcioglu M, et al. A familial outbreak of fascioliasis in Eastern Anatolia: A report with review of literatüre. Acta Trop 2011; 118: 177-183.

8. Crinquette J, Becquet R, Vilain B. Pleuro-pulmonary loeffler's syndrome of exceptional etiology. (fasciola hepatica distomatosis). J Sci Med Lille 1965; 83: 337364.

9. Rybakowska U, Pylko W, Dylikowska L. Fasciola hepatica with Loeffler's syndrome in six year old girl. Pediatr Pol 1957; 32: 173-177.

10. Akuthota P, Weller PF. Eosinophilic pneumonias. Clin Microbiol Rev 2012; 25: 649-660.

11. Bhatt NY, Allen JN. Update on eosinophilic lung diseases. Semin Respir Crit Care Med 2012; 33: 555571.

12. Long SS. Laboratory Manifestations of infectious diseases. In: Long SS, Pickering LK, Prober CG (ed). Principles and Practice of Pediatric Infectious Diseases (4th ed). China: Saunders; 2012: 1400-1412.

13. Turhan O, Korkmaz M, Saba R, Kabaaaliogu A, Inan D, Mamikoglu L. Seroepidemiology of fascioliasis in the Antalya region and uselessness of eosinophil count as a surrogate marker and portable ultrasonography for epidemiological surveillance. Infez Med 2006; 14: 208-212.

14. Zali MR, Ghaziani T, Shahraz S, Hekmatdoost A, Radmehr A. Liver, spleen, pancreas and kidney involvement by human fascioliasis: imaging findings. BMC Gastroenterol 2004; 4: 15.

15. Aksoy DY, Kerimoğlu U, Oto A, et al. Fasciola hepatica infection: clinical and computerized tomographic findings of ten patients. Turk J Gastroenterol 2006; 17: $40-45$. 